\title{
MAGNETOMETRIA IN-SITU PARA CARACTERIZAÇÃO DE MATERIAIS ELETRODEPOSITADOS
}

\author{
Vinícius C. De Franco*,a, e João E. Schmidt ${ }^{\mathrm{a}}$ \\ anstituto de Física, Universidade Federal do Rio Grande do Sul, 91501-970 Porto Alegre - RS, Brasil
}

Recebido em 24/12/2018; aceito em 24/04/2019; publicado na web em 14/05/2019

\begin{abstract}
IN-SITU MAGNETOMETRY FOR ELECTRODEPOSITED MATERIALS CHACTERIZATION. We have recently made studies of electrodeposits of Co onto graphene attached to different substrates, successfully performed via in-situ magnetization measurements. The magnetic analysis of deposits as a function of time lapse, indicate that Co grows with different magnetic characteristics from the beginning to the end of each run, independent of the substrate over which the graphene has been deposited on. In this technical note, we use these results and some obtained in several previous researches developed in our laboratory during the last 20 years, to show that the association of in-situ magnetometry with electrodeposition processes can be a powerful tool to understand the dynamics of the electrochemistry involved, as well as the physics of the magnetic materials fabricated. The Electrochemical Alternating Gradient Force Magnetometer (EC-AGFM) is appropriate for: (i) studying the magnetic properties of the electrodeposits either during deposition or dissolution; (ii) evaluating the electrodeposition dynamics via the sample's magnetic response; (iii) scrutiny of the electrodeposits as they undergo chemical changes (oxidation, etc.); (iv) studying the deposited materials from the atomic level up to bulk; v) studying the effects of addition of trace amounts of anions in the solution and their impact on the magnetic properties of metal adatoms.
\end{abstract}

Keywords: in-situ magnetometry; electrodeposition; cyclic voltammetry curves; chronoamperometry curves; $\mathrm{M} \times \mathrm{H}$ curves.

\section{INTRODUÇÃO}

Em tempos recentes, os materiais magnéticos com estruturas nanométricas têm sido utilizados em aplicações tecnológicas, como em inovadores dispositivos baseados em efeitos associados ao spin do elétron (spintrônica) ${ }^{1}$ para processamento de sinais e para armazenamento de dados ou como agregados a medicamentos na forma de nanocápsulas voltadas a liberação de drogas de forma controlada e localizada no corpo humano e também como agentes de contraste para a ressonância magnética (MRI), entre muitas outras aplicações.

Para atender às necessidades de qualquer que seja a aplicação, é necessário desenvolver materiais com estruturas ou morfologias específicas e também com propriedades magnéticas apropriadas. Dentre os processos que se utilizam para a fabricação de materiais magnéticos para estes fins, destacam-se os processos químicos ou eletroquímicos, por permitirem a obtenção dos mesmos de forma barata, rápida e com bom controle de qualidade. ${ }^{2-6}$ No entanto, usualmente, o estudo dos materiais magnéticos obtidos por estes processos demanda que inúmeras amostras (às vezes centenas) sejam obtidas e suas caracterizações magnéticas sejam realizada de forma individual e fora do sistema de produção das mesmas. Ou seja, medidas ex-situ são necessárias.

Por outro lado, a caracterização magnética de materiais nanoestruturados, seja na forma de nanocamadas ou nanopartículas, nos quais a quantidade de material magnético é exígua, exige a utilização de instrumentos de alta sensibilidade para a medição do momento magnético dos mesmos. Um instrumento que tem a sensibilidade adequada é o Magnetômetro de Gradiente de Força Magnética (AGFM), ${ }^{7-10}$ utilizado neste trabalho.

Portanto, aliando a versatilidade da capacidade de fabricação de amostras por processos químicos ou eletroquímicos com a possibilidade de caracterizar as amostras magneticamente através do AGFM, e fazendo isso simultaneamente, in-situ, é possível obter resultados do comportamento magnético dos materiais em tempo real, reduzindo a quantidade de amostras normalmente necessária para os estudos

\footnotetext{
*e-mail: 00153890@ufrgs.br
}

consistentes acima mencionados. Assim, as pesquisas ganham em eficiência e, em muitos casos, conseguem-se informações (e mesmo amostras) impossíveis de se conseguir pela abordagem tradicional.

\section{PARTE EXPERIMENTAL}

A caracterização magnética das nanoestruturas produzidas no decorrer deste trabalho foi realizada com o que denominamos de Magnetômetro Eletroquímico de Força Magnética por Gradiente Alternado (EC-AGFM).

O sistema AGFM, ${ }^{7-10}$ na sua versão original, é um instrumento sensível e apropriado para a realização de medidas de magnetização e de momento magnético de amostras micro ${ }^{7,8}$ e nanométricas em CNTP. Seu funcionamento baseia-se na força magnética $\left(\mathbf{F}_{\mathbf{M}}\right)$ que um determinado material com momento magnético (m) sofre quando submetido a um gradiente de campo magnético $\nabla \vec{H}$. Pequenas bobinas geradoras de campo magnético alternado são acopladas ao sistema, na configuração de par de Maxwell, e ligadas em série e em oposição o que maximiza o gradiente entre elas. A amostra (polarizada naturalmente ou pela ação de um eletroímã que gera um campo magnético $\mathbf{H}_{\mathrm{DC}}$ aplicado sobre ela) irá oscilar uma vez colocada entre as bobinas de gradiente, pois sofre a ação de uma força magnética $\mathbf{F}_{\mathbf{M}}$, também alternada, matematicamente descrita pela equação 1 .

$$
\vec{F}_{M}=\vec{m} \times \nabla \vec{H}
$$

Para transformar essa força em um sinal elétrico detectável e proporcional a ela, a amostra deve ser instalada no extremo inferior de uma haste constituída de um cristal piezelétrico, (conversor de sinais mecânicos, no caso de vibração da haste, em sinais elétricos) e um prolongador (no nosso caso, um tubo de vidro pirex). Esse sinal de tensão $\delta \mathrm{V}$ é proporcional a $\mathbf{F}_{\mathrm{M}}$, que também é proporcional à componente da magnetização da amostra na direção do campo $\mathbf{H}_{\mathrm{DC}}$ aplicado pelo eletroímã. $\mathrm{O} \delta \mathrm{V}$ é medido por um amplificador sensível à fase (lock-in) capaz de detectar variações de tensão da ordem de $\mathrm{nV}$, como as geradas por quantidades ínfimas de material correspondentes a ilhas de monocamadas atômicas de material magnético. ${ }^{11}$ 
Ajusta-se a frequência de oscilação do gradiente de campo magnético aos da frequência de ressonância de todo o sistema mecânico (haste/ piezoelétrico/amostra) constituinte do AGFM, buscando otimizar a razão sinal-ruído. ${ }^{11}$

Por ser um sistema capaz de monitorar sinais gerados por ilhas e, em situações limites, por monocamadas nanométricas de materiais magnéticos, cabe ressaltar que efeitos oriundos de correntes parasitas induzidas podem ser desprezados, principalmente porque tais correntes, nesse caso, anulam-se, uma vez que fluem na secção transversal perpendicular da amostra. Dentro da seção transversal, a corrente flui em ambas as direções, formando loops fechados, cancelando um ao outro devido a sua proximidade geométrica.

Em virtude de o AGFM apresentar alta sensibilidade e por apresentar a ressonância de seu sistema mecânico em faixas de frequência, no intervalo das ondas vibracionais ambientais (em torno de $18 \mathrm{~Hz}$ ), é necessário montá-lo sobre uma bancada antivibração para reduzir as interferências externas ao mesmo. Na Figura 1 é apresentado o diagrama esquemático de um AGFM semelhante ao utilizado para a obtenção dos resultados discutidos neste trabalho.

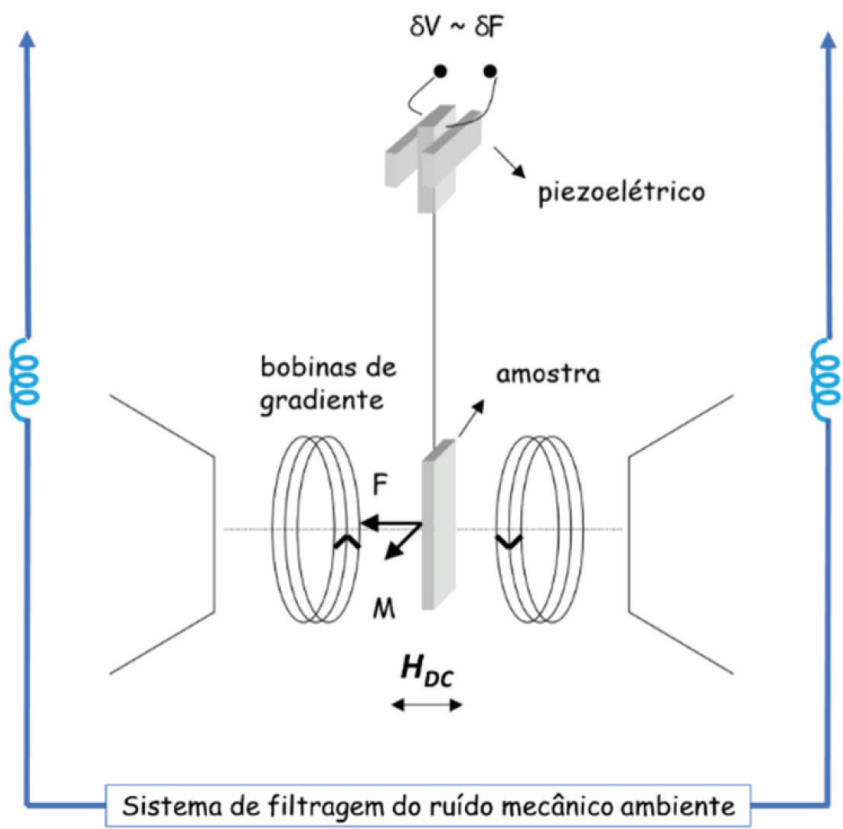

Figura 1. Diagrama do Magnetômetro de Força Magnética por Gradiente Alternado (AGFM). Adaptado de André Gündel ${ }^{11}$
Um sensor Hall é utilizado para medir o campo magnético $\mathbf{H}_{\mathbf{D C}}$ aplicado sobre a amostra e há um sistema integrado que adquire os dados automaticamente e também controla todos os demais parâmetros da medida magnética, seja ela a magnetização como função do campo aplicado ou da evolução temporal da magnetização da amostra ao longo do experimento.

\section{Magnetômetro Eletroquímico In-Situ (EC-AGFM)}

Fazendo a união das técnicas AGFM e eletrodeposição criamos o Magnetômetro Eletroquímico de Força Magnética por Gradiente Alternado (EC-AGFM). Adaptamos uma célula eletroquímica no interior dos polos do eletroímã do AGFM de fabricação caseira e também entre as bobinas de geração do gradiente de campo magnético. Uma célula em Teflon e os demais itens necessários para a realização de processos eletroquímicos foram instalados de acordo com os padrões normalmente utilizados para tal.

Os princípios físicos que regem o funcionamento do EC-AGFM (AGFM no líquido) são os mesmos que foram descritos no item anterior para o AGFM (no ar), exceto pelo fato de que agora é o eletrodo de trabalho (no qual a amostra magnética deverá ser depositada) que é colocado na parte inferior da haste de vibração e totalmente imerso na solução eletrolítica, como mostrado na Figura 2(a). O eletrodo de referência e o contra eletrodo, respectivamente de calomelano (ECS) e de platina, foram adaptados para serem colocados no interior da célula de eletrodeposição, juntamente com a entrada de argônio, gás este utilizado no processo de purga da solução eletrolítica.

$\mathrm{O}$ sistema ainda permite que se possam medir as propriedades magnéticas dos eletrodepósitos em duas configurações de campo magnético distintas: eletrodo de trabalho paralelo ou perpendicular ao campo aplicado $\mathbf{H}_{\mathbf{D C}}$, como mostrado nas Figuras 2(b) e 2(c), medidas essas importantes especialmente para o caso de depósitos na forma de filmes finos ou multicamadas, pois permite avaliar o comportamento da anisotropia magnética dos mesmos.

Com o EC-AGFM, é possível o monitoramento contínuo da evolução temporal da magnetização $(\mathrm{M} \times \mathrm{t})$, desde os estágios iniciais de depósito (a nível atômico) até o final dos processos de eletrodeposição (bulk) e após, também durante a dissolução do material previamente depositado. Em outra abordagem, é possível obter as curvas típicas de caraterização magnética, i.e. curvas de histerese magnética $(\mathrm{M} \times \mathrm{H})$, para diferentes tempos de deposição ou nos diferentes estágios da deposição. ${ }^{12}$ Isso é realizado interrompendo-se o processo de deposição em tempos específicos e obtendo, na sequência, as curvas em função do campo externo aplicado, também in-situ. Simultaneamente, a corrente eletroquímica/Faraday $(\mathrm{I} \times \mathrm{t})$; a diferença de potencial
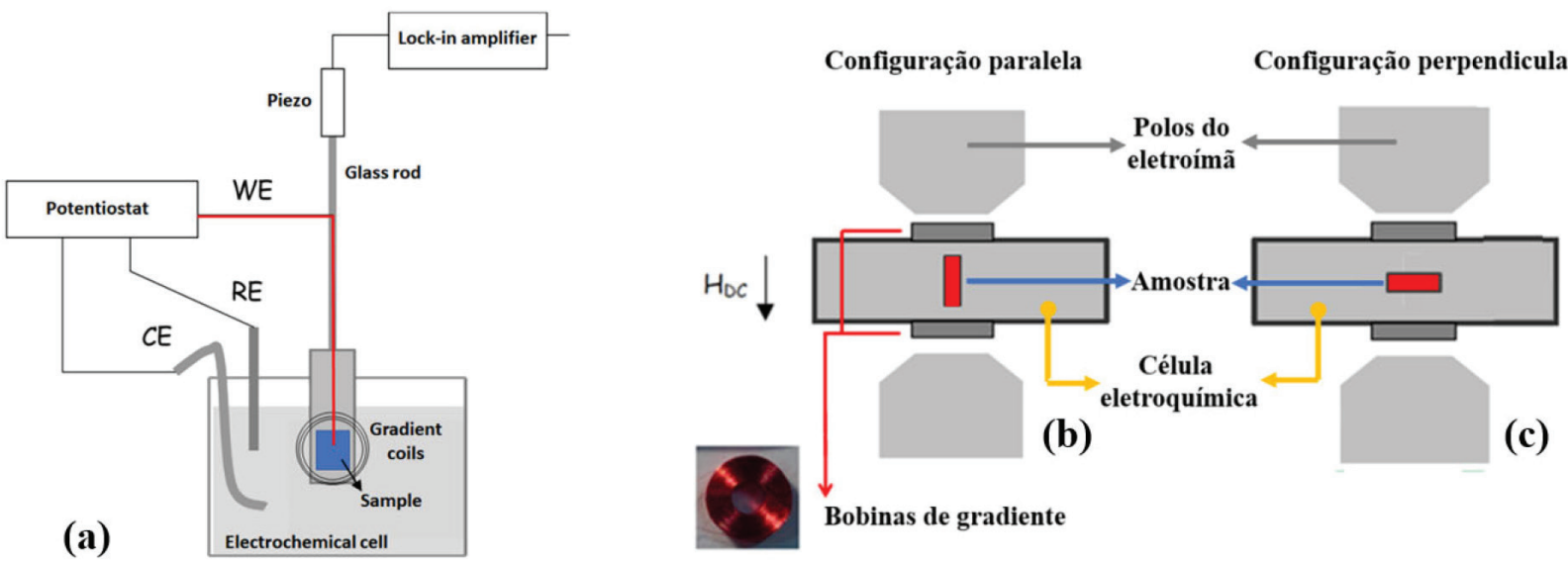

Figura 2. (a) Ilustração esquemática do EC-AGFM in-situ. Adaptado de André Gündel ${ }_{\text {; }}^{\text {(b) }}$ e (c) Disposição da amostra na configuração do sistema para medidas magnéticas com campo paralelo ou perpendicular à amostra, respectivamente 
aplicada $(\mathrm{V} \times \mathrm{t})$; e a voltametria $(\mathrm{I} \times \mathrm{V})$ em cada situação são medidas e, após, confrontadas com o comportamento das medidas magnéticas.

Importante ressaltar que os substratos a serem utilizados como eletrodo de trabalho no EC-AGFM in-situ devem ser condutores, de quaisquer tipos. A utilização do ouro como substrato deveu-se, principalmente, ao fato deste possuir uma superfície bastante estável, não sofrendo oxidação durante os processos eletroquímicos, enquanto que a utilização do grafeno teve como argumento suas excelentes propriedades físicas e químicas quando associado a outros tipos de materiais metálicos, tornando-o um material com alto potencial de estudos na área de eletroquímica. ${ }^{12}$ Vale aqui comentar que quaisquer tipos de materiais, sejam eles metais, ligas metálicas, óxidos e hidróxidos podem ser eletrodepositados utilizando-se o equipamento em questão.

O EC-AGFM montado no Laboratório de Magnetismo do Instituto de Física da UFRGS tem se revelado muito importante na caracterização de filmes ultrafinos ${ }^{13-16}$ e multicamadas ${ }^{17}$ produzidos por eletrodeposição, sendo o único no mundo com essa tecnologia in-situ. A título de exemplificar o potencial da técnica EC-AGFM, descrevemos a seguir alguns dos resultados obtidos recentemente e nos últimos anos, iniciando pelos depósitos sobre o substrato de grafeno.

\section{RESULTADOS E DISCUSSÕES}

\section{Eletrodeposição de cobalto sobre grafeno CVD}

Abaixo, os resultados obtidos recentemente com o EC-AGFM relativos às características magnéticas e eletrodinâmicas de depósitos de cobalto sobre grafeno CVD (obtido por processos de deposição química de vapor - CVD) instalado sobre substratos de $\mathrm{SiO}_{2} / \mathrm{Si}$ e cobre, e comparados com deposição sobre ouro.

Uma das funcionalidades do EC-AGFM in-situ é a possibilidade de aquisição de curvas de voltametria cíclica, utilizadas na determinação dos potenciais $U_{D E P}$ de deposição do material magnético, sem que haja a necessidade de se trocar o substrato para uma medição posterior. Na Figura 3 são apresentadas as curvas de voltametria que foram realizadas, em (a) para o grafeno $\mathrm{CVD} / \mathrm{SiO}_{2} / \mathrm{Si},{ }^{12} \mathrm{em}$ (b) para o grafeno $\mathrm{CVD} / \mathrm{Cu}$, em (c) para o $\mathrm{Au} / \mathrm{Si}(100)$ para eletrodeposição de cobalto, todas adquiridas utilizando-se a solução $\mathrm{CoSO}_{4}\left(\mathrm{SYNTH}^{\circledR}\right.$ 99\%: $\left.5 \times 10^{-3} \mathrm{~mol} \mathrm{~L}^{-1}\right)+\mathrm{H}_{2} \mathrm{SO}_{4}\left(\mathrm{SYNTH}^{\circledR} 98 \%\right.$ : $\left.1 \times 10^{-3} \mathrm{~mol} \mathrm{~L}^{-1}\right)+$ $\mathrm{K}_{2} \mathrm{SO}_{4}\left(\mathrm{MERCK}^{\circledR} 99 \%: 10^{-2} \mathrm{~mol} \mathrm{~L}^{-1}\right)+\mathrm{KCl}\left(\mathrm{MERCK}^{\circledR} 99,5 \%: 10^{-4}\right.$ mol L $\left.\mathrm{L}^{-1}\right)$ - $\mathrm{pH} \approx 3,5$, a uma velocidade de varredura do potencial de $10 \mathrm{mV} \mathrm{s}^{-1}$. O valor do $\mathrm{pH}$ em 3,5 foi ajustado adicionando-se $\mathrm{H}_{2} \mathrm{SO}_{4}$ $1 \times 10^{-3} \mathrm{~mol} \mathrm{~L}^{-1}$ à solução aquosa produzida. A escolha do $\mathrm{pH}$ em aproximadamente 3,5 se deveu a trabalhos anteriores já publicados, em que resultados bastante qualificados foram obtidos utilizando-se o $\mathrm{pH}$ ácido, por volta do valor anteriormente citado. ${ }^{11,13,15-17}$ Os substratos estavam conectados ao EC-AGFM in-situ através de um contato elétrico feito com tinta prata, livre de qualquer tipo de contaminação de origem magnética.

A partir de cada uma das voltametrias ilustradas na Figura 3, nas regiões de polarização catódica, pôde-se escolher o potencial $U_{D E P}$ para deposição potenciostática do cobalto. Para depósitos de nanopartículas ou de filmes finos, recomenda-se a escolha de um valor para $\varepsilon$ menos negativo, de baixa formação de hidrogênio e que permita um melhor controle na deposição das nanoestruturas ou dos filmes sobre o substrato. Os valores $\mathrm{U}_{\mathrm{SB}}$, denominados potenciais de stand-by, também extraídos das voltametrias ilustradas nas Figuras 3(a), 3(b) e $3(\mathrm{c})\left(\mathrm{U}_{\mathrm{SB}}=-0,6,-0,74,-0,76 \mathrm{~V}\right.$ vs. ECS, respectivamente), variam de acordo com o substrato utilizado, são os potenciais em que não ocorre nem deposição e nem dissolução de cobalto. Nestes valores, ajustados manualmente no software de controle do AGFM in-situ, que as curvas de $\mathrm{M} \times \mathrm{H}$ podem ser adquiridas após a deposição potenciostática do cobalto.

Os resultados das medidas de transientes das respostas magnéticas da deposição potenciostática de Co sobre os substratos grafeno CVD/ $\mathrm{Cu}$, grafeno $\mathrm{CVD} / \mathrm{SiO}_{2} / \mathrm{Si}$ e $\mathrm{Au} / \mathrm{Si}(100)$ (representados nas cores vermelho, preto e azul nas figuras a seguir, respectivamente), evidenciam que o cruzamento das informações extraídas das medidas magnéticas in-situ com os das medidas de densidade de corrente podem contribuir para o entendimento dos materiais eletrodepositados sobre grafeno.

Na Figura 4(a) são apresentadas as curvas de densidade de corrente normalizadas, obtidas em função do tempo de deposição potenciostática de cobalto, sobre os três substratos citados anteriormente. De acordo com as teorias da eletrodeposição, ${ }^{2,3,18}$ o comportamento padrão da densidade de corrente elétrica durante uma deposição potenciostática é semelhante ao obtido quando o $\mathrm{Au} / \mathrm{Si}(100)$ foi utilizado como substrato. Esse comportamento pode ser descrito pelo modelo de Scharifker e Hills: ${ }^{18}$ inicialmente, a formação de núcleos de crescimento do depósito aumenta a área eletroativa do eletrodo, aumentando o módulo da densidade de corrente de deposição até o ponto em que a área eletroativa se torna máxima, correspondente ao pico de corrente de deposição (momento de máxima nucleação). Após o estabelecimento desta área máxima, formam-se zonas isoladas em que a deposição ocorre pelo transporte de massa no eletrólito. A superfície eletroativa tende a diminuir e ocorre decréscimo no módulo da densidade de corrente. O regime estacionário é atingido quando as zonas isoladas de difusão coalescem e ocupam toda interface entre o eletrodo e o eletrólito.

Porém, chama a atenção nesses resultados, o comportamento anômalo da densidade de corrente elétrica durante a eletrodeposição do cobalto sobre substratos à base de grafeno CVD (curvas em vermelho e em preto), que não se encaixa nos modelos tradicionais que explicam a eletrodeposição de materiais metálicos sobre substratos condutores. Essa anomalia também tem manifestação consistente na resposta magnética.
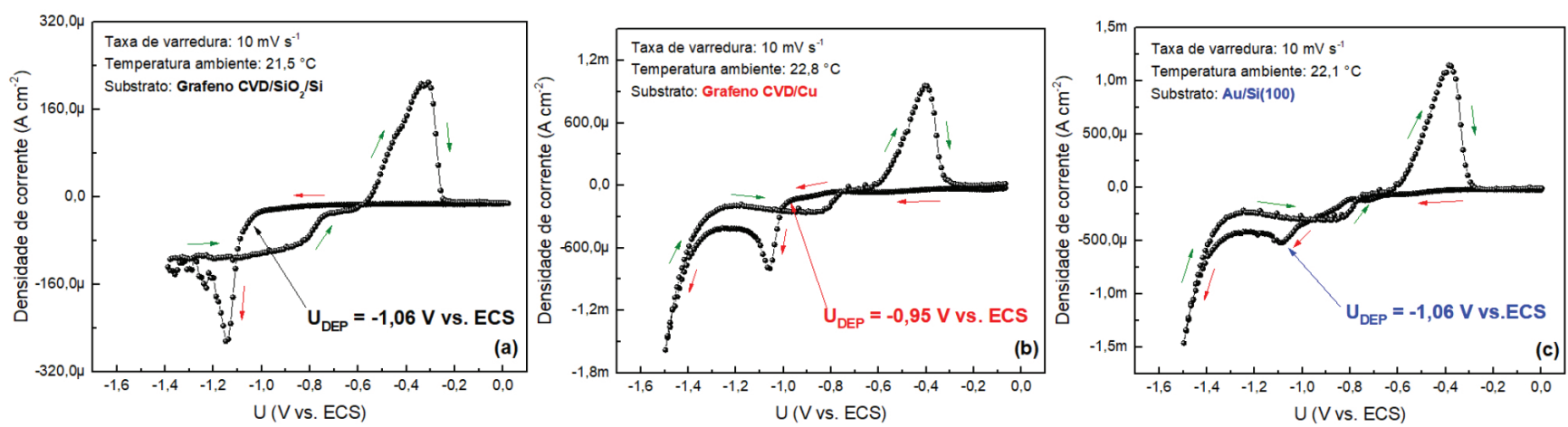

Figura 3. Voltametria cíclica obtida para o (a) grafeno $\mathrm{CVD} / \mathrm{SiO} / \mathrm{Si}$, (b) grafeno $\mathrm{CVD} / \mathrm{Cu}$, (c) $\mathrm{Au} / \mathrm{Si}(100)$, em solução de sulfato de cobalto com $\mathrm{pH} \approx 3,5$, em varredura a uma taxa de $10 \mathrm{mV} \mathrm{s}^{-1}$ 

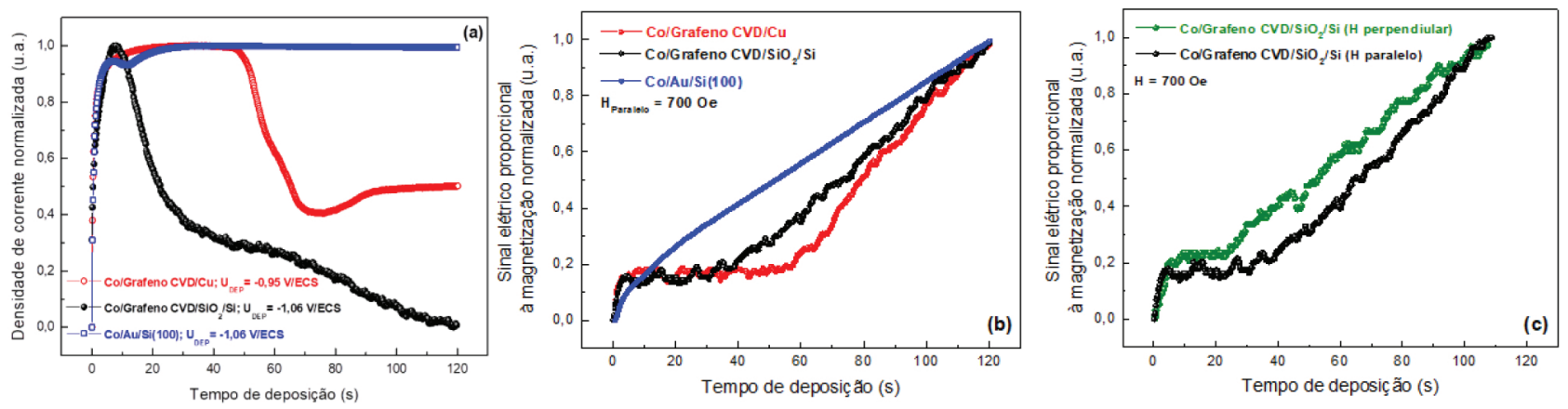

Figura 4. (a) Curvas de densidade de corrente normalizada em função do tempo de depósito potenciostático de cobalto sobre grafeno CVD/Cu (vermelho), grafeno $\mathrm{CVD} / \mathrm{SiO} / \mathrm{Si}$ (preto) e Au/Si(100) (azul); (b) transientes de resposta magnética normalizada do cobalto eletrodepositado potenciostaticamente sobre grafeno $\mathrm{CVD} / \mathrm{Cu}$ (vermelho), grafeno $\mathrm{CVD} / \mathrm{SiO} \mathrm{O}_{2} / \mathrm{Si}$ (preto) e Au/Si(100) (azul), com campo de 700 Oe aplicado paralelamente à superfície do substrato; (c) comparação entre os transientes de resposta magnética do cobalto eletrodepositado potenciostaticamente sobre grafeno CVD/SiO $/ 2 \mathrm{Si}$, com campo de 700 Oe aplicado em configuração perpendicular (verde) e paralelo (preto) ao substrato

Na Figura 4(b) tem-se os transientes de resposta magnética normalizada do cobalto eletrodepositado potenciostaticamente sobre grafeno $\mathrm{CVD} / \mathrm{Cu}$, grafeno $\mathrm{CVD} / \mathrm{SiO}_{2} / \mathrm{Si}$ e $\mathrm{Au} / \mathrm{Si}(100)$ (curvas vermelha, preta e azul), com campo de 700 Oe aplicado paralelamente à superfície do substrato. Observa-se a presença de um rápido crescimento da magnetização, durante aproximadamente $2 \mathrm{~s}$, seguido de uma estabilização no valor da magnetização, que acaba por formar um platô (magnetização constante) em ambas as curvas obtidas. O platô não é observado quando o substrato utilizado foi o $\mathrm{Au} / \mathrm{Si}(100)$, indicando que esta parece ser uma característica específica de materiais magnéticos eletrodepositados em substratos à base de grafeno CVD. A extensão da duração do platô não é a mesma para a deposição de Co sobre o substrato de grafeno CVD/ $\mathrm{Cu}$ e sobre o grafeno $\mathrm{CVD} / \mathrm{SiO}_{2} / \mathrm{Si}$, uma vez que os potenciais $\mathrm{U}_{\mathrm{DEP}} \mathrm{de}$ deposição utilizados são ligeiramente diferentes, mesmo utilizando-se a mesma solução eletrolítica. A extensão temporal do platô sobre grafeno $\mathrm{CVD} / \mathrm{SiO}_{2} / \mathrm{Si}$ teve duração de, aproximadamente, $25 \mathrm{~s} \mathrm{(1 \textrm {s } <}$ $\mathrm{t}<26 \mathrm{~s}$ ), enquanto que a duração referente ao substrato grafeno CVD/ $\mathrm{Cu}$ foi de $55 \mathrm{~s}(1 \mathrm{~s}<\mathrm{t}<56 \mathrm{~s})$.

A resposta magnética dos depósitos sobre ouro (em azul) segue um comportamento esperado, ou seja, que ela seja proporcional à quantidade de material depositado e dependente da morfologia e/ou da estrutura cristalina do depósito, mas ferromagnética. Nas duas curvas referente aos depósitos sobre o grafeno (em preto e vermelho), a resposta magnética acompanha a anomalia apresentada na densidade de corrente, indicando claramente que há três evoluções magnéticas bastante diferenciadas para todo o processo de deposição. Inicialmente, o material depositado apresenta resposta com características ferromagnéticas $(0-2 \mathrm{~s})$ e, na sequência, o material deixa de ter variação mensurável da resposta magnética, pois permanece constante (entre 2 e aproximadamente 26 ou $56 \mathrm{~s}$ ), indicando que o material depositado pode estar sendo oxidado (por exemplo, $\mathrm{Co}_{2} \mathrm{O}_{3}$ é paramagnético à temperatura ambiente) e, após, o sinal volta a ter características ferromagnéticas com o sinal aumentando proporcional à quantidade de material depositado.

Foi levantada a hipótese de que a constância do sinal magnético no platô, medida na configuração paralela, estaria relacionada com a alteração da anisotropia do material depositado e não devido à sua oxidação. Para verificar essa possibilidade, o EC-AGFM permite realizar o mesmo experimento instalando o substrato grafeno $\mathrm{CVD} / \mathrm{SiO}_{2} / \mathrm{Si}$ paralelo e perpendicular ao campo aplicado (preto e verde na Figura 4(c), respectivamente) e os resultados mostram que não há alteração do sinal magnéticos neste intervalo em ambos os casos. Portanto, não há evolução da anisotropia do material de planar para perpendicular na região do platô.

Esse exemplo mostra a profusão de novas informações e questionamentos que podem ser extraídos do uso do EC-AGFM, mostrando que a técnica é muito útil para quem trabalha com materiais magnéticos eletrodepositados, especialmente os nanoestruturados. Estudos adicionais devem levar à interpretação precisa dos fenômenos físicos e químicos que justifiquem o comportamento dos mesmos frente os dados originais acima arrolados.

Influência na anisotropia perpendicular dos átomos da periferia de ilhas de monocamadas de Co depositados sobre $\mathrm{Au}^{19}$

Nesse estudo, "Edge atoms effects on the perpendicular anisotropy of ultrathin magnetic layers", ${ }^{19}$ constatou-se que a magnetização medida através do EC-AGFM, em função do tempo do eletrodepósito de camadas ultrafinas de $\mathrm{Co} / \mathrm{Au}(111)$ (ver Figura 5), apresentaram dois picos de fácil magnetização para fora do plano da amostra.

Esses resultados foram explicados vinculando a resposta magnética aos átomos da periferia das ilhas de Co sobre ouro, que crescem camada por camada atômica e apresentam alta anisotropia magnética perpendicular $\left(\mathrm{M}_{\perp}\right)$ ao plano da amostra. Isso mostra que é possível obter materiais que apresentem alta anisotropia magnética em grãos muito pequenos, mesmo abaixo do limite paramagnético, abrindo oportunidades para aplicações tecnológicas através da engenharia dos materiais.

\section{Deposições potenciostáticas de multicamadas de $\mathrm{Cu}-\mathrm{Co}$}

No estudo da eletrocristalização de multicamadas magnéticas $\mathrm{Cu}-\mathrm{Co}$, amplamente utilizadas em sistemas magnetoresistivos, Günde $1^{11}$ utilizou o EC-AGFM in-situ na confecção e análise magnética do referido sistema multicamada. Com o objetivo de se melhor compreender a mecânica de crescimento destas multicamadas, a técnica de eletrodeposição pulsada, com campo magnético em configuração paralela aplicado, com eletrólito único, foi utilizada. Os substratos utilizados foram o $\mathrm{Au}(111)$ e o vidro ITO.

As medidas de magnetização in-situ de filmes multicamadas durante o processo de eletrodeposição de multicamadas de $\mathrm{Co}-\mathrm{Cu},{ }^{17}$ seja no modo transiente, seja no modo do levantamento das curvas de histerese para tempos específicos de deposição (ver Figura 6), revelou que foi possível avaliar eficiência do aumento da magnetização em função da carga utilizada no processo. Essa eficiência tende a decrescer na medida em que o número de bicamadas aumenta, provavelmente devido ao aumento da rugosidade da superfície em formação. As multicamadas apresentaram comportamento ferromagnético típico, com a magnetização e o campo coercivo aumentando conforme o número de bicamadas e/ou a espessura da camada de cobalto foram aumentadas. 


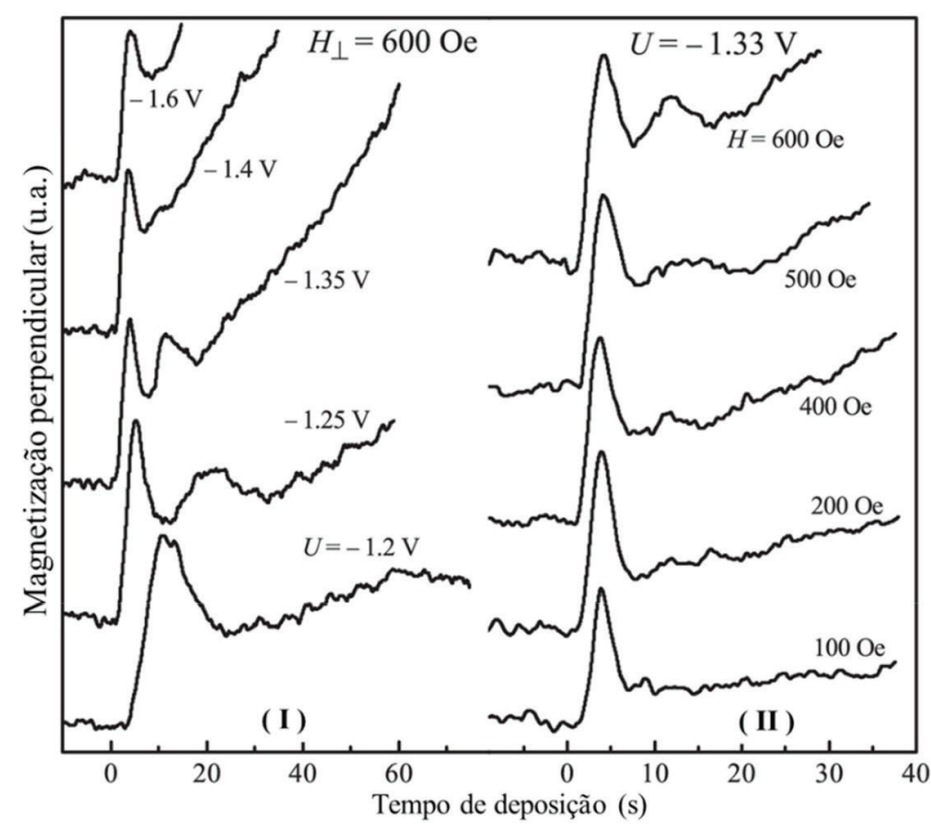

(a)
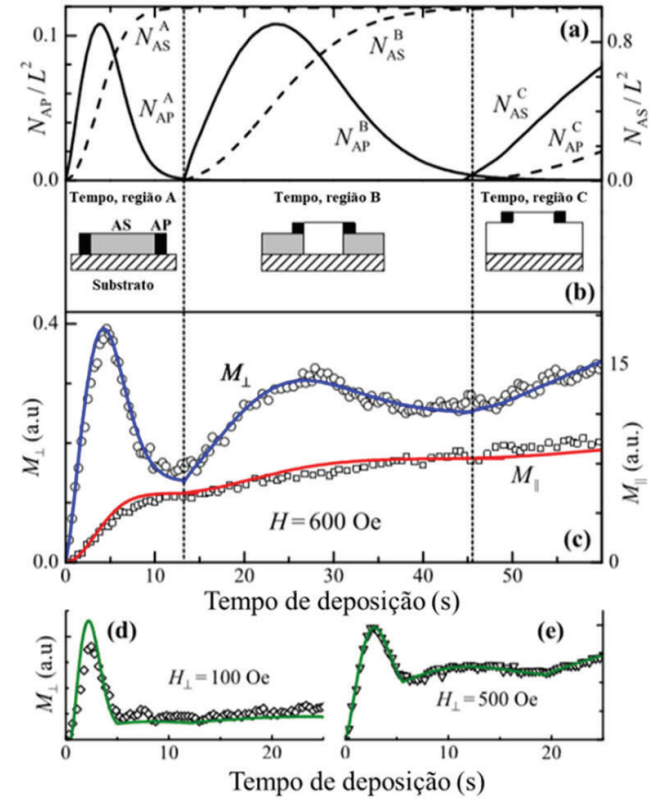

(b)

Figura 5. Reproduzida da referência 19, com a permissão da AIP Publishing (a-I) Transientes de $M_{\perp}$ ( $($ ) gravados “in-situ” durante a eletrodeposição de Co para um campo fixo de $H=600$ Oe e variando o potencial de depósito $U$; (a-II) Transientes variando H para o potencial de depósito constante $U_{\text {DEP }}=-1,33$; (b) Resultados das simulações teóricas do comportamento da magnetização perpendicular devido à contribuição dos átomos da periferia das ilhas de cobalto: para detalhes ver referência 19
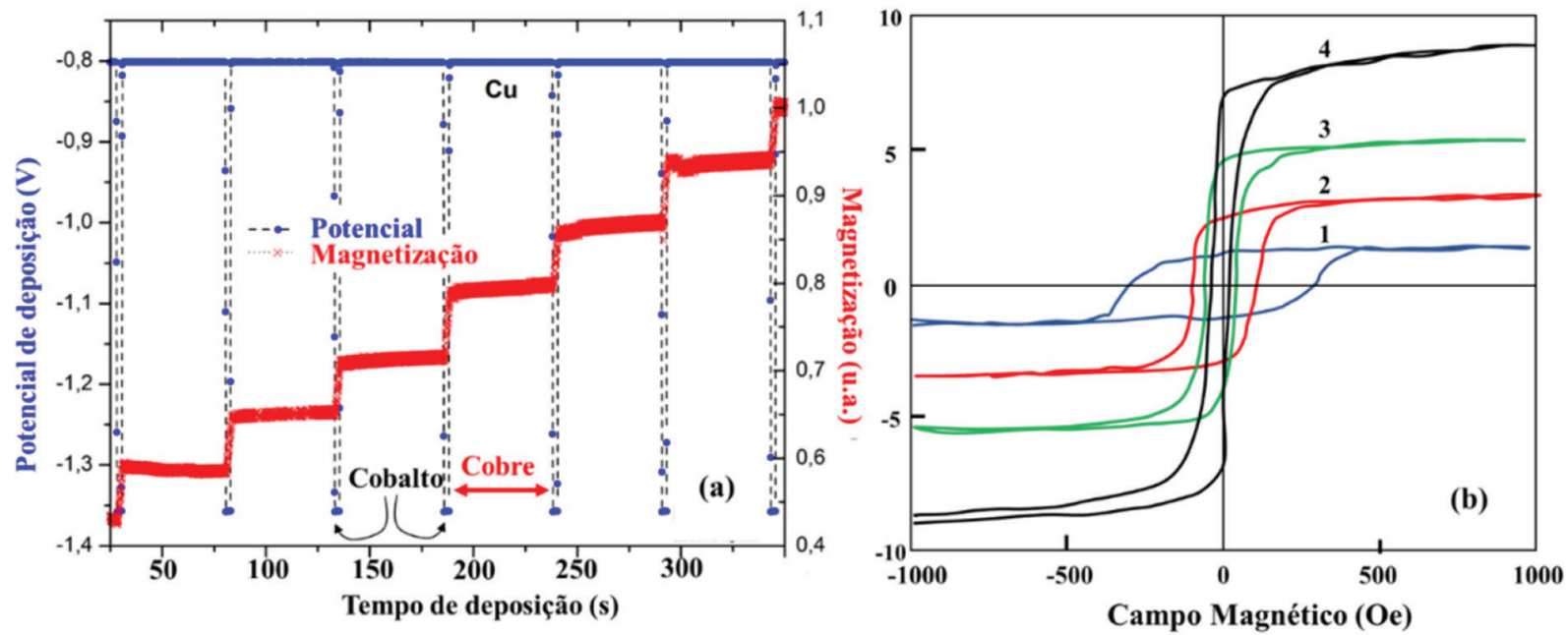

Figura 6. Reproduzida e adaptada da referência 17, com a permissão da AIP Publishing (a) Curvas de potencial elétrico e transiente da resposta magnética da deposição intercalada de bicamadas de Co/Cu sobre vidro ITO. Os seis pulsos de deposição variaram entre $U_{C o}=-1,35 \mathrm{~V}$ vs. MSE e $U_{C u}=-0,8 \mathrm{~V}$ v. MSE; (b) Curvas de histerese obtidas “in-situ” após a deposição de [Co(13nm)/Cu(11nm) para 1 bicamada (curva b1); 2,3 e 4 bicamadas (curvas b2, b3 e b4)

\section{Medidas magnéticas in-situ de camadas eletrodepositadas ultrafinas de $\mathrm{Co}$, $\mathrm{Ni}$ e $\mathrm{Fe} / \mathrm{Au}(111) / \mathrm{Mica}^{15}$}

Neste trabalho foram medidas propriedades magnéticas no EC-AGFM na interface eletroquímica de um processo de crescimento de amostras de metais 3d, com poucas monocamadas atômicas (ML), sobre substrato de Au/Mica. Foi possível fazer uma avaliação da anisotropia magnética dos depósitos em função do transiente temporal do processo e os resultados mostraram fortes diferenças entre as interfaces $\mathrm{M} / \mathrm{Au}(111)$, com $\mathrm{M}=\mathrm{Co}, \mathrm{Ni}$ e Fe. Foi constatado que o eixo de fácil magnetização é planar para as amostras de níquel, independente da espessura dos filmes, enquanto que é perpendicular ao plano para cobalto e ferro para espessuras abaixo de um valor crítico $t^{*}\left(\approx 1-2 \mathrm{ML}\right.$ e 2-3 ML, respectivamente). Acima de $t^{*}$, a direção de fácil magnetização gira gradualmente para o plano das amostras como pode ser constatado na Figura 7.

\section{CONCLUSÃO}

Como alegado inicialmente, neste artigo é demonstrado, com dados originais recentes e com exemplos de resultados anteriores obtidos em nossos laboratórios, que é possível obter informações magnéticas e eletroquímicas relevantes e diferenciadas através da técnica que foi desenvolvida no Laboratório de Magnetismo do Instituto de Física da UFRGS denominada de Magnetômetro Eletroquímico de Força Magnética por Gradiente Alternado (EC-AGFM). Ela permite que se avalie o comportamento do processo eletroquímico e a resposta magnética de materiais depositados simultaneamente, in-situ. A 
(a)

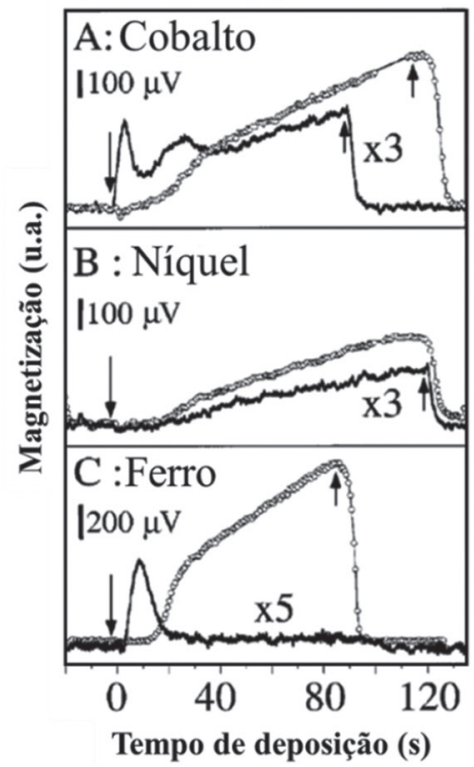

(b)

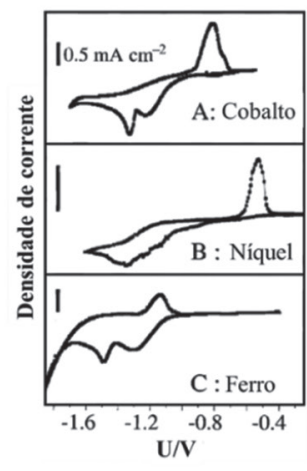

(c)

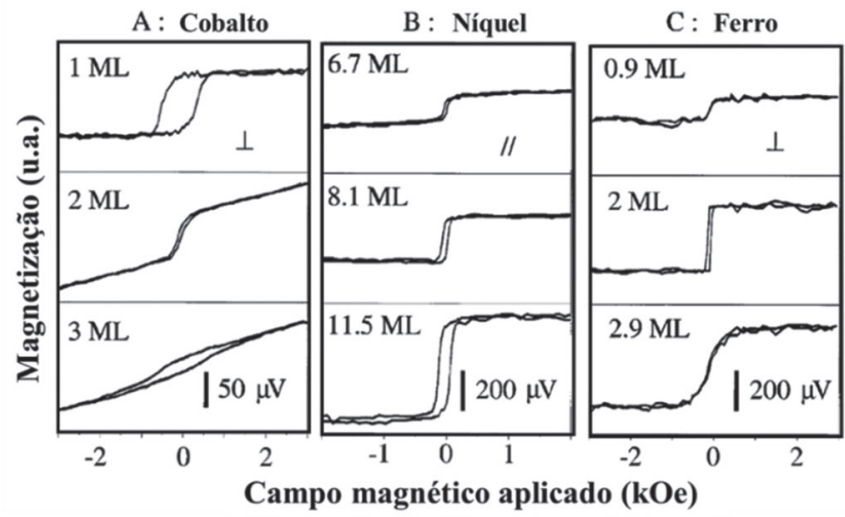

Figura 7. Reproduzida da referência 15 com a permissão da PCCP Owner Societies (a) Transientes "in-situ" M(t) sob campo de 600 Oe, obtidos durante a eletrodeposição dos metais sobre $\mathrm{Au}(111)$. (A) Cobalto, $U_{D E P}=-1,3 \mathrm{~V} ;$ (B) níquel. $U_{D E P}=-1,4 \mathrm{~V}$; (C) Ferro, $U_{D E P}=-1,5 \mathrm{~V}$. Dados foram adquiridos no modo paralelo (// - no plano-símbolos vazios) e no modo perpendicular ( $\perp$ - símbolos cheios). As setas $\uparrow$ e $\downarrow$ indicam o início de deposição e o início do "stripping". A notação $x 3$ ou $x 5$ indica a diferença de escalas entre as medidas. (b) Voltametria do substrato Au(111)/mica realizada na célula do EC-AGFM e em configuração perpendicular para soluções de cobalto $(A)$, níquel $(B)$ e ferro $(C)$. A taxa de verredura foi de $50 \mathrm{mV} \mathrm{s}^{-1}$. Ver o texto original para a composição das soluções. ${ }^{15}$ (c) Laços de histereses magnéticas adquiridos "in-situ" para cobalto, níquel e ferro sobre Au(111). A configuração do sistema para as medidas foram no modo perpendicular para o Fe e o cobalto e no plano para o níquel. As espessuras indicadas são em monocamadas (ML) e foram extraídas do método de "stripping". Os potenciais de depósitos foram os mesmos da Figura (a) e os potencias de interrupção dos depósitos para a realização das medidas foram $U=-1,15+/-0,02 \mathrm{~V}$ (cobalto e níquel) ou $U=-1,32+/-0,02 \mathrm{~V}$ (ferro)

técnica é suscetível a adaptações para também ser utilizada na análise de materiais obtidos por simples reações químicas. Portanto, a técnica de magnetometria in-situ para caracterização de materiais pode ser útil para diversos ramos da pesquisa científica e tecnológica, sejam eles vinculados à Química, Física ou Ciência e Engenharia dos Materiais.

\section{REFERÊNCIAS}

1. Fert, A.; George, J.-M.; Jaffres, H.; Faini, G.; J. Phys. D: Appl. Phys. 2002, 35, 2443.

2. Alper, M. Em Electrodeposition of Multilayered Nanostructures; Shi, D., Aktaş, B., Pust, L., Mikailov, F., eds.; Springer-Verlag: Berlin, 2002, cap. 7.

3. Compton, R. G.; Sanders, G. H. W.; Electrode Potentials, Oxford University Press: Oxford, 1996.

4. González, J. T. C.; Tese de Doutorado, Universidad Central de Venezuela, Venezuela, 2009.

5. Rocha, A. S.; Tese de Doutorado, Universidade Federal de Santa Catarina, Brasil, 2006.

6. Dorico, E.; Tese de Doutorado, Universidade Federal de Santa Catarina, Brasil, 2010.
7. Zijlstra, H.; Review on Scientific Instruments 1970, 41, 1241.

8. Roos, W.; Hempel, K. A.; Voight, C.; Dedericks, H.; Schippan, R.; Review of Scientific Instruments. 1980, 51, 612.

9. Flanders, P. J.; J. Appl. Phys. 1998, 63, 3940.

10. Flanders, P. J.; Review of Scientific Instruments 1990, 61, 839.

11. Gündel, A.; Tese de Doutorado, Universidade Federal do Rio Grande do Sul, Brasil, 2002.

12. De Franco, V. C.; Castro, G. M. B.; Corredor, J.; Mendes, D.; Schmidt, J. E.; Carbon Letters 2017, 21, 16.

13. Cagnon, L.; Devolder, L.; Cortes, R.; Morrone, A.; Schmidt, J. E.; Chappert, C.; Allongue, P.; Phys. Rev. B. 2001, 63, 104419.

14. Gündel, A.; Morrone, A.; Schmidt, J. E.; Cagnon, L.; Allongue, P.; J. Magn. Magn. Mater. 2001, 226, 1616.

15. Gündel, A.; Cagnon, L.; Gomes, C.; Morrone, A.; Schmidt, J. E.; Allongue, P.; Phys. Chem. Chem. Phys. 2001, 3, 3330.

16. Cagnon, L.; Gündel, A.; Devolder, T.; Morrone, A.; Schmidt, J. E.; Allongue, P.; Appl. Surf. Sci. 2000, 164, 22.

17. Gündel, A.; Chassaing, E.; Schmidt, J. E.; J. Appl. Phys. 2001, 90, 5257.

18. Scharifker, B.; Hills, G.; Electrochim. Acta 1983, 28, 879.

19. Geshev, J.; Gündel, A.; Zaharieva, I.; Schmidt, J. E.; Appl. Phys. Lett. 2012, 101, 132407. 